VOL. V, FAsc. 1 E 2, P, 189-199, 6 PL.

\title{
ESPECIES NOVAS DE HARPACTICOIDA (COPEPODA - CRUSTACEA) ENCONTRADAS EM ALGAS MARINHAS DO LITORAL PARANÁ - SANTA CATARINA \\ (RECEOIDO EM 22/VII/84)
}

\section{Hans Jakobi}

Seogão de Zoologia - Instituto de Pesquisas da Universidade do Paraná

Êste trabalho liga-se diretamente aos estudos hidro-faunísticos publicados no ano passado, revelando formas desconhecidas de Harpacticoida da zona de arrebentação do litoral Paraná-Santa Catarina. Se bem que a totalidade dos Harpacticoida que ocupam êste biótopo ainda não pode ser avaliado, até agora já me foi possível encontrar 10 espécies e 2 subespécies novas no referido habitat. Quatro dessas espécies novas pertencem a 3 gêneros diferentes $e$ novos para a Ciência (compare-se Jakobi, Dusenia IV (1) 1953 e Dusenia IV (2) 1953). Nêste trabalho são descritas as seguintes formas: Scutellidium loureiroi n.sp. (denominado em homenagem ao Exmo.Prof.Dr. José Loureiro Fernandes, Diretor do Instituto de Pesquisas da Universidade do Paraná), Alteu tha rara n.sp., Lourinia arma ta sulamericana n. subsp., Ka tacletodes disproportionatus n. gen. n.sp. (Katacletodes por ser um gênero da família Cletodidae, improportionatus por ser o $\mathrm{P}_{\mathrm{s}}$ maior que $\mathrm{P}_{1}$ e êste muito reduzido em comparação ao comprimento total do corpo), Onychocamptus besnardi n.sp. (denominado em homenagem ao Exmo.Prof. W. Besnard, Diretor do Instituto Oceanográfico, da Univ. de S.Paulo), He terolaophon te manifera sulamericana n. subsp., He terolaophonte hamatus (hamatus segmento genital com 2 cerdas em forma de gancho).

Consigno os meus agradecimentos ao Instituto de Pesquisas da Faculdade de Filosofia da Universidade do Paraná assim como ao Conselho Nacional de Pesquisas pelo auxílio prestado a êste trabalho. 


\title{
DESCRIÇÃO DAS FORMAS NOVAS
}

\author{
Ord. COPEPODA \\ Sub-ord. PODOPLEA \\ Super-fam. HARPACTICOIDA \\ Fam. TISBIDAE
}

\author{
Scutellidium Claus 1866 \\ Psamathe Philippi 1840, p. 189. \\ Cantocamptus kroeyer 1863, p. 408. \\ Stephenson 1913, p. 355. \\ Scutellidium Brady 1880a, p. 175. \\ Claus 1889, p. 23. \\ Machairopus Brady 1883, p. 104. \\ Lang 1936a, p. 35. \\ Idya Brian 1920 , p. 30. \\ Idyaea Brian $1923 b$, p. 129. \\ Tisbe Gurney 1927d, p. 496. \\ willey 1930, p. 83.
}

Scutelḷidium loureiroi n. sp.

(P1. I, Figs. 1-14)

DESCRICQÃO DA FEMEA - Cefalotórax enorme, de forma oval. Comprimento do abdômen igual ao conjunto dos 3 últimos segmentos rorácicos. Segmento genital dorso-lateral dividido. Rostrum relativamente grande com cerdas delgadas na margem, base não separada da fronte.

$A_{1}$ : 9-articulada; proporção dos artículos igual a Scutellidium longicauda. As três cerdas terminais também articuladas.

$A_{2}$ : Exp. 4- (às vêzes 5-) articulado com 3 cerdas terminais bem diferentes.

$P_{1}$ : Exp. alcança o limite entre o $1^{\circ}$ e $2^{\circ}$ artf́culo do Enp. $02^{\circ}$ artf́culo do Enp. com uma cerda interna cuja parte basal é bem alargada. 0 espinho da parte externa da base é extremamente grande e largo na parte proximal.

$\mathrm{P}_{2}-\mathrm{P}_{4}$ : Tôdas as cerdas são articuladas.

$\mathrm{P}_{\mathbf{s}}$ : Forma geral igual a Scutellidium longicauda; a localização 
e proporções das cerdas valem como caracteres distintivos (compare-se a fig. correspondente).

TAManho - 0,8 - 0,9mm.

ECOLOGIA - Dois exemplares na folhagem das algas Endocladia (Chlorophyceae) e Codium (Siphonales), fixadas nas pedras existentes em lugares periòdicamente cobertos pela água do mar da praia de Itapocoroi (Santa Catarina).

Macho desconhecido.

Localizagão e número das cerdas

\begin{tabular}{|l|l|l|l|l|l|l|l|l|l|l|l|l|l|l|l|l|l|}
\hline \multicolumn{3}{|c|}{$\mathrm{P}_{2}$} & \multicolumn{5}{c|}{$\mathrm{P}_{3}$} & \multicolumn{5}{c|}{$\mathrm{P}_{4}$} \\
\hline \multicolumn{2}{|c|}{ Exp. } & \multicolumn{3}{|c|}{ Enp. } & \multicolumn{3}{|c|}{ Exp. } & \multicolumn{2}{|c|}{ Enp. } & \multicolumn{3}{|c|}{ Exp. } & \multicolumn{2}{c|}{ Enp. } \\
\hline 1 & 1 & 323 & 1 & 2 & 321 & 1 & 1 & 323 & 1 & 2 & 321 & 1 & 1 & 323 & 1 & 2 & 221 \\
\hline
\end{tabular}

Fam. PELTidiIdAe Sars

Alteutha Baird $1845 a$

Cyclops Baird 1887, p. 809.

Irpacticus Uilno-Edwards 1840, Vol. III, p. 480.

Sterope Goodsir 1845, p. 826.

Carillus Goodsir 1845, p. 826.

Peltidium Brady 1880a, p. 158.

Poppo $1885 a$, p. 191.

Eupe lte Claus 1889, p. 12.

Canu 1892, p. 150.

Ilteutha Claus 1898 , p. 6 .

Sars 1904a, p. 61.

Alteutha rara n. sp.

(P1. II, Figs. 1-11)

DESCRIÇAO DO MACHO - Corpo oval, segmentos torácicos nos limites dorsais não serrilhados. Rostrum bem saliente, recurvado para baixo. Furca em forma de duas lâminas providas de 5 cerdas cada uma.

$A_{1}$ : 7- ou 8-articulado, com estetascos no $3^{\circ}$ e $5^{\circ}\left(6^{\circ}\right)$ segmento. Hapl b́cero, úl timo segmento com gancho terminal. 
$A_{2}$ : Exp. 2-articulado, o $1^{\circ}$ artículo com duas, o $2^{\circ}$ com três cerdas. Base e $1^{\circ}$ artículo Enp. com uma cerda respectivamente.

Md. : Com Ex. e Enp., sendo êles bem separados.

$P_{1}$ : Artículo terminal do Exp. com três cerdas e uns espinhos, o Exp. isento de cerdas na margem interna. Enp. também 3-articulado, $1^{\circ}$ artículo com uma, $2^{\circ} \mathrm{sem}, 3^{\circ}$ artículo com duas cerdas na margem interna respectivamente.

$\mathrm{P}_{5}$ : Enp. bem reduzido, Exp. com uma cerda e dois apêndices terminais. Exemplares jovens ainda têm o Enp. separado da cerda terminal, enquanto os adultos apresentam os dois reunidos.

Tamanho $-0,7-0,8 \mathrm{~mm}$.

ECOLOGIA - Foram encontrados 3 machos nas algas das pedras situadas diante da fortaleza - Ilha do Mel (Báa de Paranaguá).

Fêmea desconhecida.

Localização e número das cerdas

\begin{tabular}{|c|c|c|c|c|c|c|c|c|c|c|c|c|c|c|c|c|c|}
\hline \multicolumn{6}{|c|}{$\mathrm{P}_{2}$} & \multicolumn{6}{|c|}{$\mathrm{P}_{3}$} & \multicolumn{6}{|c|}{$P_{4}$} \\
\hline \multicolumn{3}{|c|}{ Exp. } & \multicolumn{3}{|c|}{ Enp. } & \multicolumn{3}{|c|}{ Exp. } & \multicolumn{3}{|c|}{ Enp. } & \multicolumn{3}{|c|}{ Exp. } & \multicolumn{3}{|c|}{ Enp. } \\
\hline 0 & 1 & 223 & 1 & 2 & 221 & 0 & 1 & 323 & 1 & 2 & 221 & 0 & 1 & & 1 & 2 & 221 \\
\hline
\end{tabular}

Fam. LOURINIIDAE Monard

Lourinia Wilson 1924

Jurina n.gen. Claus 1866, p. 24.

Ceylonia n.gen. Thompson \& Scott 1903, p. 285.

Ceyloniella wilson 1924, p. 14.

Lourinia armata Claus

Lourinia armata sulamericana n. subsp.

(PI. III, Figs. 1-17)

DESCRIÇÃO DA FEMEA - Corpo cil ́́ndrico, com constrição no limite do $4^{\circ}$ - $5^{\circ}$ segmento torácico. A largura do abdômen diminue paulatinamente para trás. Todos os segmentos abdominais providos de espinhos na parte dorso-lateral. Cefalotórax mais comprido que os três segmentos seguintes. Rostrum saliente, com duas cerdas delgadas mas resistentes, incubadas em duas elevações do rostrum. Labium enorme, pubescente na parte frontal. Furca duas vêzes mais comprida que larga com uma só cerda bem desenvolvida, não pubescente e pelo menos de igual tamanho que o abdômen. 
Md. : Placa com cinco dentes e uma parte lateral provida de uma cerda serreada. Exp. pequeno, mas claramente separado da base, com 3 cerdas.

$A_{1}$ : 7-articulada, estetasco no $4^{\circ}$ artículo. Os primeiros dois artículos são largos e bem resistentes.

$A_{2}$ : Alobase com Exp. pequeno em forma de uma lâmina, com duas cerdas terminais. Enp. na parte externa especialmente quitinizada, com espinhos grandes.

$P_{1}-P_{4}$ : Expos. 3-articulados, Enpos. 2-articulados. Ponte com dois suportes cruzados. $2^{\circ}$ artículo Exp. $\mathrm{P}_{2}$ sem cerda na margem interna.

$P_{s}$ : Exp. e Enp. em forma de duas placas enormes, com sulcos que conduzem às cerdas. Benp. um pouco maior que Exp., a maioria dos exemplares com 5 cerdas, sendo a mais interna a menor e a segunda de fora para dentro sempre menor que a mais externa. Encontrei também indivíduos com 6 cerdas no Benp., limitando-se a variaçâo à parte interna do Benp. (veja-se a figura). Exp. sempre com 5 cerdas, sendo a da parte apical maior que as outras que são iguais entre si.

TAMANHO - $1 \mathrm{~mm}$.

DESCRICÃ̃O DO MACHO - Forma do corpo e proporção de segmentos como na fêmea. Enp. $\mathrm{P}_{3}$ 2-articulado, sendo a base do $2^{\circ}$ artículo transformado numa parte bem quitinizada com apófise que é mais comprida que o Enp. inteiro. Quero destacar que o Enp. não é 3-articulado conforme os desenhos e descrições de outros autores (Claus, Thompson, Scott), embora a parte quitinosa, sem dúvida, dê uma tal impressão; além disso a forma da apófise difere notàvelmente das até agora conhecidas, e é de côr dourada.

$P_{5}$ : Benp. e Exp. reunidos formando uma só placa.

$P_{s}^{s}:$ Em forma de saliência provida de três cerdas.

TAMANHo - 0,9mm, de côr azul intenso, igual à fêmea.

ECOLOGIA - 6 fêmeas e 3 machos nas algas Endocladia (Chlorophyceae) e Codium (Siphonales) existentes na zona das marés da praia de Santa Catarina e Paraná.

OBSERVAÇ̃̃E - A faḿllia Louriniidae baseia-se numa só espécie até agora conhecida. Não duvido de que existam pelo menos três espécies diferentes (compare-se fig. 490, p. 1216, em Lang: Monographie der Harpakticiden). Entretanto deixei de destacar as formas por mim encontradas como espécie nova por não ter material para comparar. Chama-se a atenção para o fato que, futuramente, o Enp.P, com a apófise deve dar um caráter essencial para a distinção nítida de espécies. 
Localizagão e número das cerdas

\begin{tabular}{|l|l|l|l|l|l|l|l|l|l|l|l|l|l|l|l|l|l|}
\hline \multicolumn{5}{|c|}{$P_{2}$} & \multicolumn{5}{c|}{$P_{3}$} & \multicolumn{5}{c|}{$P_{4}$} \\
\hline \multicolumn{3}{|c|}{ Exp. } & \multicolumn{3}{|c|}{ Enp. } & \multicolumn{3}{c|}{ Exp. } & \multicolumn{3}{|c|}{ Enp. } & \multicolumn{3}{c|}{ Exp. } & \multicolumn{3}{c|}{ Enp. } \\
\hline 0 & 0 & 123 & 1 & 524 & - & 1 & 1 & 123 & 1 & 224 & - & 0 & 1 & 123 & 1 & 123 & - \\
\hline
\end{tabular}

Fam. CleTOdIDAE Scott

Katacletodes n.gen.

DIAGNOSE - Corpo comprido, cabeça apenas um pouco mais larga que os segmentos do tórax. Segmento genital dorsalmente dividido. Úl timo segmento abdominal evidentemente menor que qualquer um dos segmentos do corpo. A, 5-articulado com o $3^{\circ}$ artículo bem forte. $A_{2}$ com alobase que tem uma cerda na parte interna. Exp. com 4 cerdas. $01^{\circ}$ artículo do Enp. $P$, maior que $2^{\circ}$, isento de cerdas. Exp. 3-articulado, sendo duas cerdas bem plumosas na parte apical. Os primeiros e segundos artículos dos Expos. $P_{1}-P_{5}$ isentos de cerdas na margem interna. Úl timos artículos dos Expos. $\mathrm{P}_{2}-\mathrm{P}_{4}$ com uma cerda na margem interna localizada bem no ápice. Enpos. $P_{1}$ e $P_{4}$ são 2-articulados, os dos $\mathrm{P}_{2}-\mathrm{P}_{3}$ l-articulados. Segmento genital com duas elevações providas de duas cerdas cada uma.

GENOTIPO - Katacletodes improportionatus n.sp.

Katacletodes improportionatus n. sp.

$$
\text { (P1. IV, Figs. 1-11) }
$$

DESCRICÃO DA FEMEA - 0 corpo cil índrico e de côr parda até incolor. Todos os segmentos torácicos e abdominais são ornamentados. Segundo e terceiro segmentos abdominais com uma elevação (apêndice) em cada lado, providos de cerdas e espinhos. Furca mais comprida que larga com uma cerda não pubescente e bem reforçada. Opérculo em forma de meia-lua e espinhosa.

$A_{1}$ : 5-articulada, o $3^{\circ}$ artículo bem forte e provido de um espinho no lado externo, estetasco no $4^{\circ}$ artículo.

$A_{2}$ : Com espinhos e cerdas na parte apical do Enp. -Exp. com 4 cerdas não pubescentes e de tamanho igual.

$P_{1}$ : Enp. menor que Exp. Base e protopódito reduzido.

$\mathrm{P}_{2}$ : Enp. 1-articulado, estreito, com uma cerda na margem interna e uma apical não pubescente. Exp. quase três vêzes mais comprido que Enp. com espinhos fortes. 
$\mathrm{P}_{3}$ : Formas do Enp. e Exp. correspondem no essencial ao $\mathrm{P}_{2}$. Enp. também com uma cerda na margem interna e uma apical não pubescente. Além disso existe na parte externa uma cerda lisa de colocação entre a la. e $2 a$. cerdas anteriormente mencionadas.

P : Enp. 2-articulado, mais largo que os Enpos. correspondentes aos $\mathrm{P}_{2}-\mathrm{P}_{3}$. A cerda apical é reduzida a um espinho. Margem interna provida de duas cerdas pequenas.

$P_{5}$ : Muito grande em comparação ao $P_{1}-P_{4}$. Benp. maior que Exp. (i.é o contrário a $P_{1}-P_{4}$ ). Enp. com 3 cerdas na margem interna e 2 terminais. Exp. nf́tidamente separado da base e do Enp., com 6 oerdas, sendo as 3 mais externas pubescentes.

TAManho - 0,9mm.

ECOLOGIA - 5 indivíduos nas algas marinhas da praia de Santa Catarina e 7 indivíduos em biótopos correspondentes da praia de Guaratuba.

$$
\begin{aligned}
& \text { Localizacão e número das cerdas em comparação } \\
& \text { a outros gêneros da família CLRTODIDAE }
\end{aligned}
$$

\begin{tabular}{|c|c|c|c|c|c|c|c|c|c|c|c|c|c|c|c|c|c|c|}
\hline \multirow[b]{3}{*}{ No. so articulo } & \multicolumn{6}{|c|}{$P_{2}$} & \multicolumn{6}{|c|}{$P_{3}$} & \multicolumn{6}{|c|}{ P. } \\
\hline & \multicolumn{3}{|c|}{ Ene. } & \multicolumn{3}{|c|}{ Eno. } & \multicolumn{3}{|c|}{ Exp. } & \multicolumn{3}{|c|}{ Fnp. } & \multicolumn{3}{|c|}{ Eno. } & \multicolumn{3}{|c|}{ Enp. } \\
\hline & 1 & 2 & 3 & 1 & 2 & 3 & 1 & 2 & 3 & 2 & 2 & 3 & 1 & 2 & 3 & 1 & 2 & 3 \\
\hline 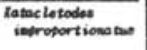 & 0 & 0 & 113 & 110 & - & - & 0 & 0 & 113 & 211 & - & - & 0 & 0 & 113 & 0 & 210 & - \\
\hline Aontosoliteos ap. & 0 & 0 & 222 & 010 & - & - & 0 & 0 & 122 & 020 & - & - & 0 & 1 & 222 & 010 & - & $\cdot$ \\
\hline Henciclotodet ap. & 0 & 0 & 223 & 1 & 121 & - & 。 & 0 & 323 & 1 & 221 & - & 0 & 323 & - & 1 & 121 & - \\
\hline Soyliclatodes ap. & 0 & 0 & 022 & 0 & 020 & - & 0 & 2 & 222 & 0 & 021 & - & 0 & 1 & 222 & 0 & 021 & - \\
\hline Leroulutroeceses ap. & 0 & 0 & 022 & 0 & 011 & - & 0 & $\circ$ & 222 & ○ & 221 & - & $\circ$ & 0 & 222 & 0 & 121 & - \\
\hline
\end{tabular}

Fam. LAOPHONTIDAE Scott

Heterolaophonte Lang 1948

Cyclops Baird 1834, p. 87; 1837, p. 380 (part.).

Canthocamptus Baird 1845, p. 154.

Baird 1850, p. 204 (part.).

Cleta Norman 1868, p. 256.

Czerniavaki 1868, p. 42.

Dacty lopusia Normann 1908b, p. 388 (part.). Laophonte Autores. 


\section{Heterolaophonte manifera Wilson \\ Heterolaophonte manifera sulamericana n. subsp.}

$$
\text { (P1. V, Figs. 1-8) }
$$

DESCRIÇÃO DA FEMMEA - Cefalotórax e os três segmentos torácicos seguintes bastante mais largos que o $5^{\circ}$ segmento torácico e os segmentos abdominais em conjunto. Rostrum saliente e estreito. Furca apenas pouco mais comprida que larga, com duas cerdas terminais bem desenvolvidas. Exp. A completamente desaparecido, no local ainda duas cerdas bem delgadas. Este caráter é o principal para justificar esta subespécie. Heterolaophonte manifera Wilson ainda possue um Exp. embora já muito reduzido.

$\mathrm{P}_{1}-\mathrm{P}_{5}$ : Igual a H.manifera.

TAMANHO $-0,5 \mathrm{~mm}$.

DESCRIÇÃO DO MACHO - Corpo igual ao da fêmea. Os caracteres principais do $\mathrm{P}_{2}-\mathrm{P}_{3}$ descritos por Wilson valem também para estas formas. Especialmente a forma da cerda na margem interna do Enp. $P_{2}$ é caracterìsticamente alargada no meio. É notável que o Enp. $\mathrm{P}_{1}$ as vêzes parece 3-articulado pelo fato de que a parte do protopódito que se liga ao Enp. tem a tendência de se separar do resto do protopódito. Portanto, concluo que esta parte do protopódito, característico para a famflia Laophontidae, representa o $1^{\circ}$ artículo Enp. P, normalmente sempre reunido com o protopódito.

TAMANHO $-0,5 \mathrm{~mm}$.

ECOLOGIA - 11 machos e 2 fêmeas, colhidas em algas (Rhodophyceae e Chlorophyceae) existentes nas pedras periòdicamente cobertas pelas águas das marés em Itapocoroi e Porto Belo (Santa Catarina).

Heterolaophonte hamatus n.sp.

$$
\text { (P1. V, Figs. 9-12) }
$$

DESCRIÇÃO DA FEMEA - Corpo da fêmea aerodinâmico; furca pouco mais comprida que larga, com 2 cerdas terminais bem desenvolvidas. A, 7-articulado, sendo os 3 primeiros artículos bem mais largos que os 4 seguintes. $A_{2}$ com alobase bem larga, Exp. reduzido a uma pequena elevação no devido local.

$P_{1}$ : Enp. na margem interna com 7-8 espinhos fortes. Protopódito igualmente com uma série de espinhos.

$\mathrm{P}_{2}-\mathrm{P}_{4}$ : Veja-se tabela sôbre o número e localização das cerdas. $\mathrm{P}_{\mathrm{s}}$ : Exp. com 6, Enp. com 5 cerdas.

TAMANHO $-0,5 \mathrm{~mm}$. 
ECOLOGIA - 7 fêmeas e um macho nas algas afixadas nas pedras em Itapocoroi e Porto Belo (Santa Catarina).

Localização e número das cerdas no gênero HETEROLAOPHONTE

\begin{tabular}{|c|c|c|c|c|c|c|c|c|c|c|c|c|c|}
\hline \multirow{3}{*}{\multicolumn{2}{|c|}{\begin{tabular}{|c|}
$P_{n}$ \\
No. dos artífulos
\end{tabular}}} & \multicolumn{4}{|c|}{$\mathrm{P}_{2}$} & \multicolumn{4}{|c|}{$P_{3}$} & \multicolumn{4}{|c|}{$\mathrm{P}_{4}$} \\
\hline & & \multicolumn{2}{|c|}{ Exp. } & \multicolumn{2}{|c|}{ Enp. } & \multicolumn{2}{|c|}{ Exp. } & \multicolumn{2}{|c|}{ Enp. } & \multicolumn{2}{|c|}{ Exp. } & \multicolumn{2}{|c|}{ Enp. } \\
\hline & & 2 & 3 & 1 & 2 & 2 & 3 & 1 & 2 & 2 & 3 & 1 & 2 \\
\hline \multirow{2}{*}{\multicolumn{2}{|c|}{$\begin{array}{l}\text { Heteroleophonts } \\
\text { hamatus n. ap. }\end{array}$}} & & & & & & & & & & & & \\
\hline & & 1 & 123 & 0 & 220 & 1 & 123 & 0 & 321 & 1 & 022 & 0 & 121 \\
\hline Grupo & "Stroomi" & 1 & 123 & 0 & 220 & 1 & 123 & 0 & 321 & 1 & 123 & 0 & 121 \\
\hline$"$ & "Vinuta" & 1 & 123 & 0 & 220 & 1 & 123 & 0 & 321 & 0 & 022 & 0 & 121 \\
\hline " & "Littoralis" & 1 & 123 & 0 & 220 & 1 & 123 & 0 & 221 & 1 & 023 & 0 & 121 \\
\hline . & "Quinquespinosa" & 1 & 123 & 0 & 120 & 1 & 223 & 0 & 221 & 1 & 223 & 0 & 211 \\
\hline$"$ & "Oampbelliensis" & 1 & 123 & 0 & 220 & 1 & 223 & 0 & 321 & 1 & 223 & 0 & 121 \\
\hline$"$ & "Temusispina" & 1 & 023 & 0 & 220 & 1 & 023 & 0 & 321 & 1 & 023 & 0 & 121 \\
\hline$"$ & "Discophora" & 1 & 123 & 0 & 120 & 0 & 123 & 0 & 121 & 0 & 023 & 0 & 120 \\
\hline
\end{tabular}

Onychocamptus Daday 1903, p. 157

Laophonte Autores (part.).

Cleta vários Autores.

Onychocamptus besnardi n. sp.

(PI. VI, Figs. 1-15)

DESCRIÇÃO DA FEMEA - Cefalotórax quase redondo; rostrum saliente, igualmente redondo. O corpo estreita-se paulatinamente para trás. Furca duas vêzes mais comprida que larga, com 5 cerdas, sendo a mais externa subdividida em três artículos. A cerda apical é a maior e tem uma incisura na parte proximal.

$A_{1}$ : 5-articulada, estetasco no $3^{\circ}$ artículo.

$A_{2}$ : Exp. 1-articulado, com 4 cerdas, sendo duas na margem interna e duas terminalmente colocadas.

$P_{1}$ : Enp. e Exp. 2-articulados, $1^{\circ}$ artículo Enp. sem qualquer cerda. Ul timo artículo Exp. com 5 cerdas constantemente. 
$\mathrm{P}_{2}-\mathrm{P}_{4}$ : Os primeiros artículos dos Enpos. sempre faltos de qualquer cerda. $03^{\circ}$ artículo Enp. $\mathrm{P}_{4}$ com duas cerdas na margem externa. Pernas em geral pouco quitinizadas, hialinas.

$P_{5}$ : Exp. em larga distância do Benp. Ambos com três cerdas. A parte externa da base onde se insere a cerda externa, é inchada de tal maneira que o $P_{5}$ em conjunto é trilobuloso. A estrutura de $\mathrm{P}_{5}$ e demais caracteres demonstram um certo parentesco com Onychocamptus bengalensis Sewell 1934, encontrado em água salobra perto de Calcuttá (fndia).

TAMANHO - 0,6mm.

DESCRIÇÃO DO MACHO - Forma geral, especialmente a do cefalotórax, igual à da fêmea. As duas elevações laterais no $2^{\circ}$ e $3^{\circ}$ segmento abdominal assim como a cerda típica do lado externo da furca identificam o macho indubitàvelmente como pertencendo à espécie Onychocamptus besnardi. $\mathrm{P}_{3}$ é modificado: Enp. 3-articulado, sendo $\circ 3^{\circ}$ bem reforçado na margem externa, terminando num espinho. $02^{\circ}$ artículo na margem interna com uma cerda curvada (gancho bem quitinizado).

TAMANHO - 0,3mm - exatamente a metade do tamanho da fêmea adulta (não de fêmeas em copulação).

ECOLOGIA - 15 fêmeas e 7 machos em algas marinhas e substrato areno-lodoso da costa Santa-Catarina-Paraná.

Loca 1 ização e número das cerdas no gênero ONYCHOCAMPTUS

\begin{tabular}{|c|c|c|c|c|c|c|c|c|c|c|c|c|}
\hline \multirow[b]{3}{*}{ No. do artículo } & \multicolumn{4}{|c|}{$P_{2}$} & \multicolumn{4}{|c|}{$P_{3}$} & \multicolumn{4}{|c|}{$P_{4}$} \\
\hline & \multicolumn{2}{|c|}{ Exp. } & \multicolumn{2}{|c|}{ Enp. } & \multicolumn{2}{|c|}{ Exp. } & \multicolumn{2}{|c|}{ Enp. } & \multicolumn{2}{|c|}{ Exp. } & \multicolumn{2}{|c|}{ Enp. } \\
\hline & 2 & 3 & 2 & 2 & 2 & 3 & 1 & 2 & 2 & 3 & 1 & 2 \\
\hline Anychocans tus & & & & & & & & & & & & \\
\hline besnardi n. ap. & 1 & 123 & 0 & 220 & 1 & 123 & 0 & 221 & 1 & 022 & 0 & 111 \\
\hline Grupo "Hohamred" & 1 & 123 & 0 & 220 & 1 & 123 & 0 & 321 & 1 & 123 & 0 & 211 \\
\hline " "Horricus" & 1 & 123 & 0 & 220 & 1 & 223 & 0 & 220 & 1 & 222 & 0 & 120 \\
\hline$"$ "Kliei" & 0 & 023 & 0 & 120 & 0 & 023 & 0 & 121 & 0 & 023 & 0 & 121 \\
\hline
\end{tabular}




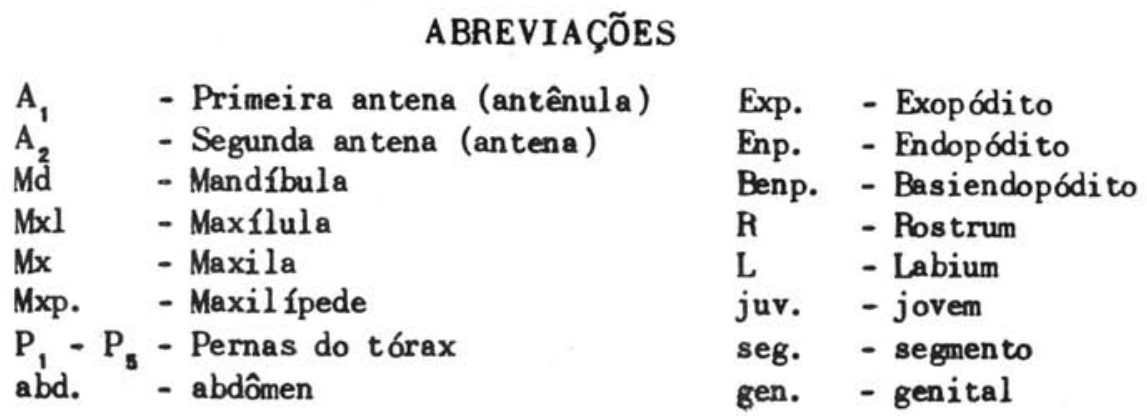

\section{RES U MO}

O autor, estudando Harpacticoida (Copepoda - Crustacea) provenientes da zona de arrebentação do litoral dos Estados Paranś-Sta.Catarina(Brasil) apresenta nêste trabalho 1 gênero, 3 espécies e 2 subesptoies novas para a ciência. A investigação decorre de estudos hidro-faunisticos que o autor vem efetuando sistematicamente no litoral sul brasileiro, achando-se ilustrado o trabalho com 6 estampas contendo $7 \theta$ desenhos olucidativos.

\section{S U M M A R Y}

Continuing the series of investigations about Harpacticoida (Copepoda(rustacea) found in sea-weeds of the breakers' region of southern Brazil (Paran and Santa Catarina) the author describes in this paper 1 genus, 3 species and 2 subspecies as new for science. The study is illustrated by 6 plates with 79 figures.

B I B L I OGRA F I A

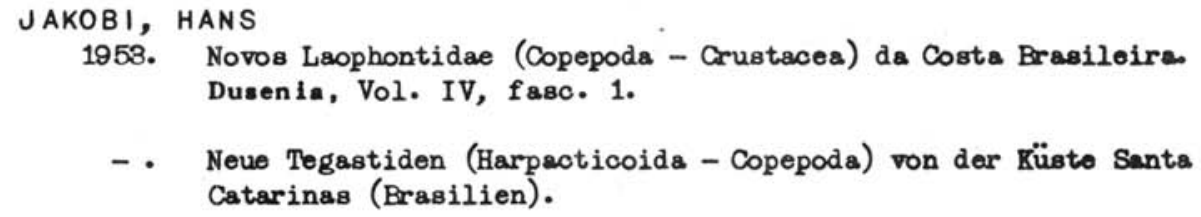

LANG, KARL

1948. Monographie der Harpakticiden. 2 vol., Lund.

WILSON, C.B.

1932. The Copepod Crustaceans of Chesapeake Bay. Proc.U.S.Nat.Mus., Vol. 80 . 
ESTAMPA I

\section{Scutellidium loureiroin.sp.}

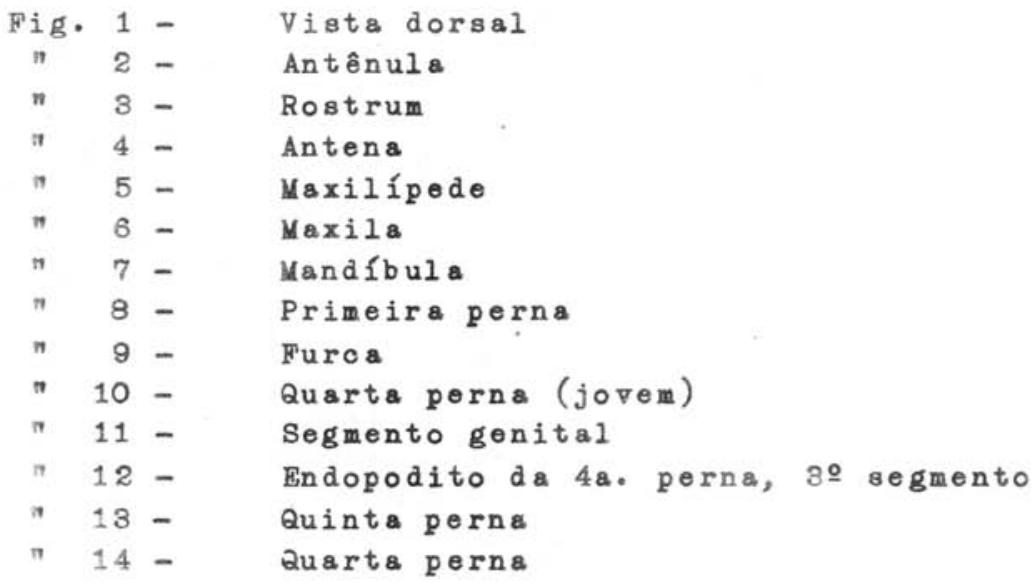



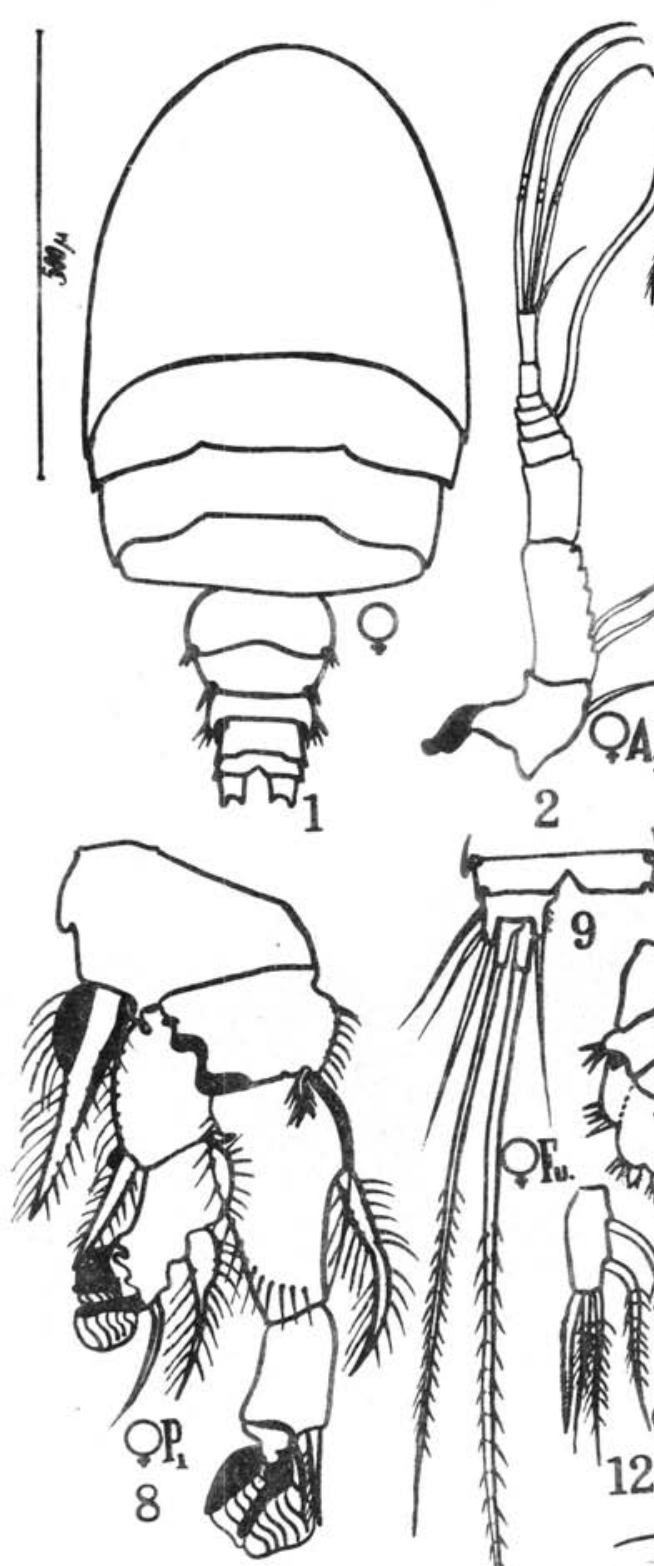

2
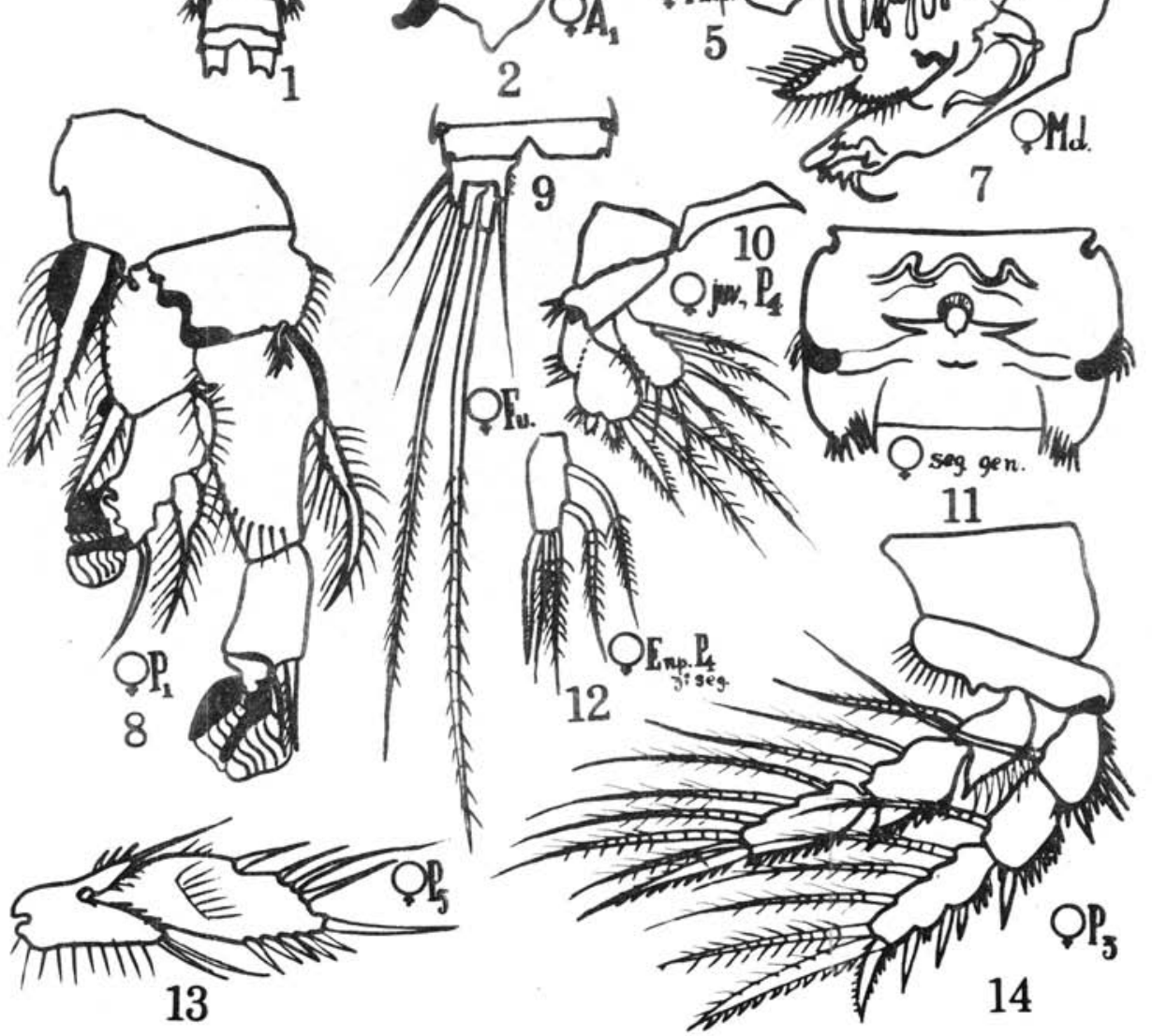


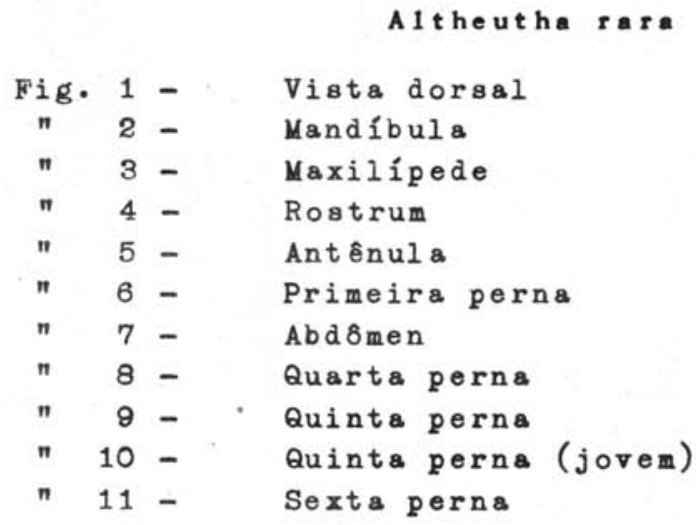

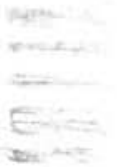


JAkоB, H. - Espécies novas de Harpacticoida...

ESTAMPA II
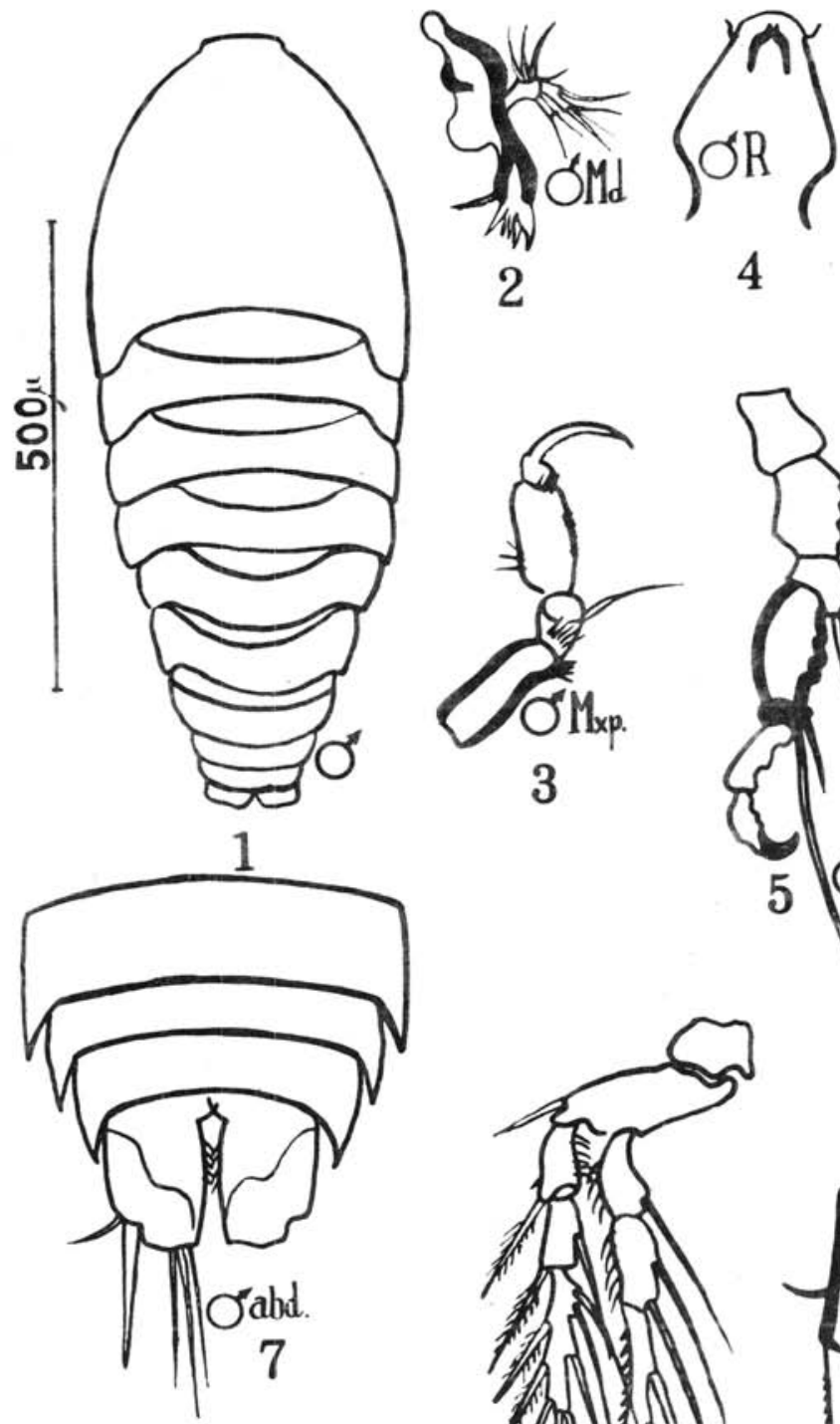

2

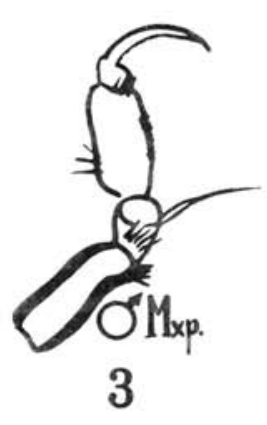

4
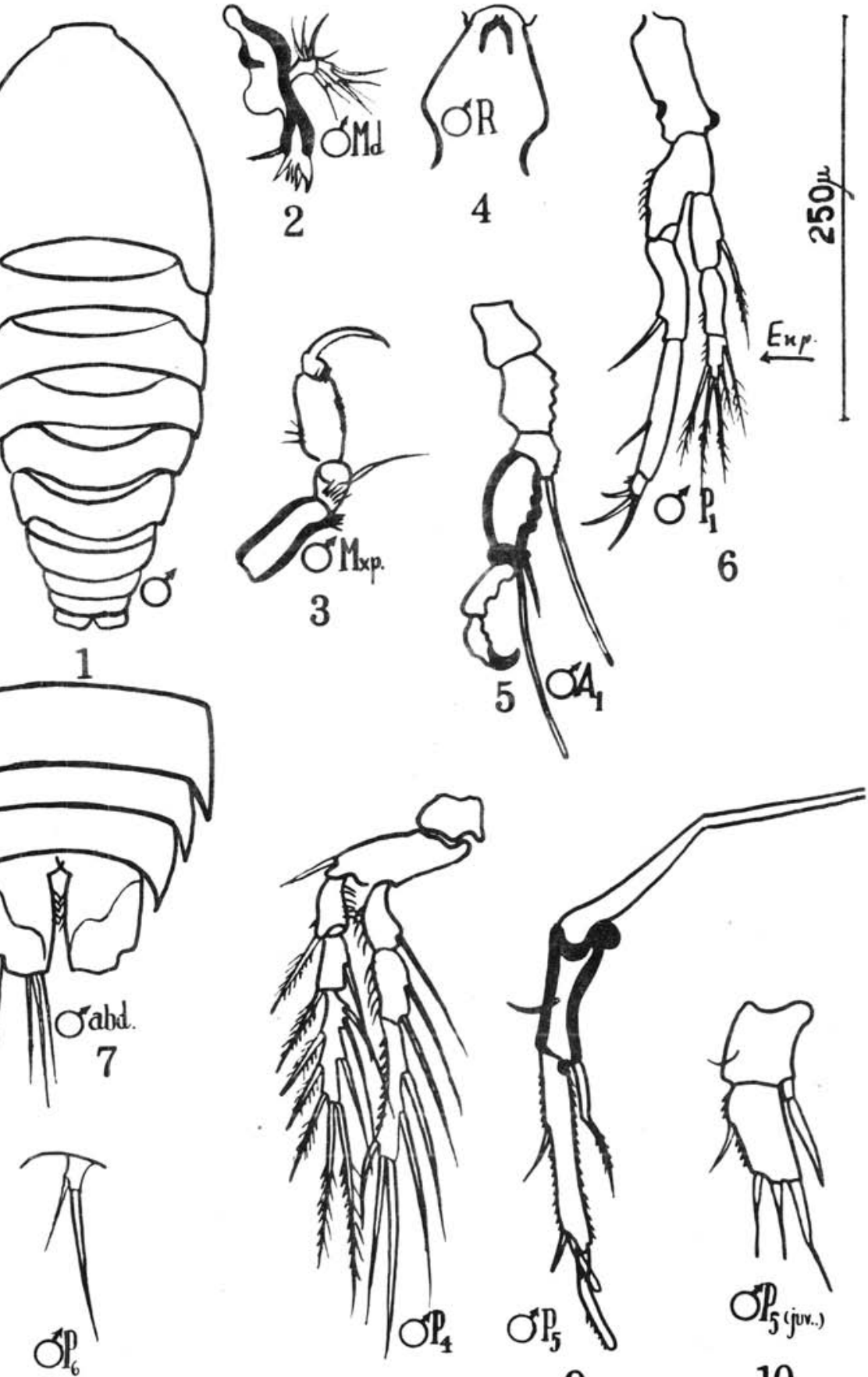

8

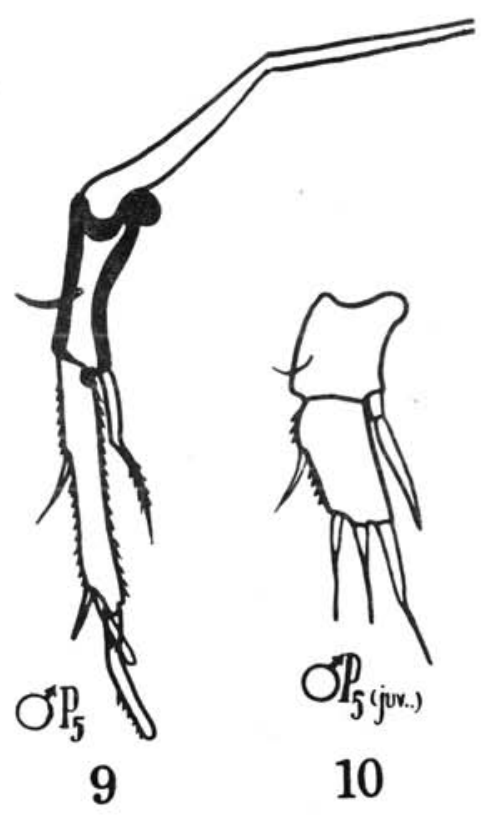


Lourinia amata ulamericana nosubsp.

\begin{tabular}{|c|c|c|c|}
\hline Fig. & 1 & - & Vista dorsal \\
\hline " & 2 & - & Rostrum \\
\hline$\pi$ & 3 & - & Labrum \\
\hline * & 4 & - & Ant ênula \\
\hline$n$ & 5 & - & Antena \\
\hline$"$ & 6 & - & Mandíbula \\
\hline$\pi$ & 7 & - & Maxila \\
\hline$n$ & 8 & - & Primeira perna \\
\hline$"$ & 9 & - & Segunda perna \\
\hline$n$ & 10 & - & Teroeira perna \\
\hline " & 11 & - & Quarta perna \\
\hline$n$ & 12 & - & Quinta perna \\
\hline$n$ & 13 & - & Segmento genital \\
\hline$n$ & 14 & - & Ant ênula \\
\hline$\pi$ & 15 & - & Terceira perna \\
\hline$\pi$ & 16 & - & Quinta perna \\
\hline$\pi$ & 17 & - & Sexta perna \\
\hline
\end{tabular}


Jakobi, H. - Espécies novas de Harpacticoida...

ESTAMPA I I I
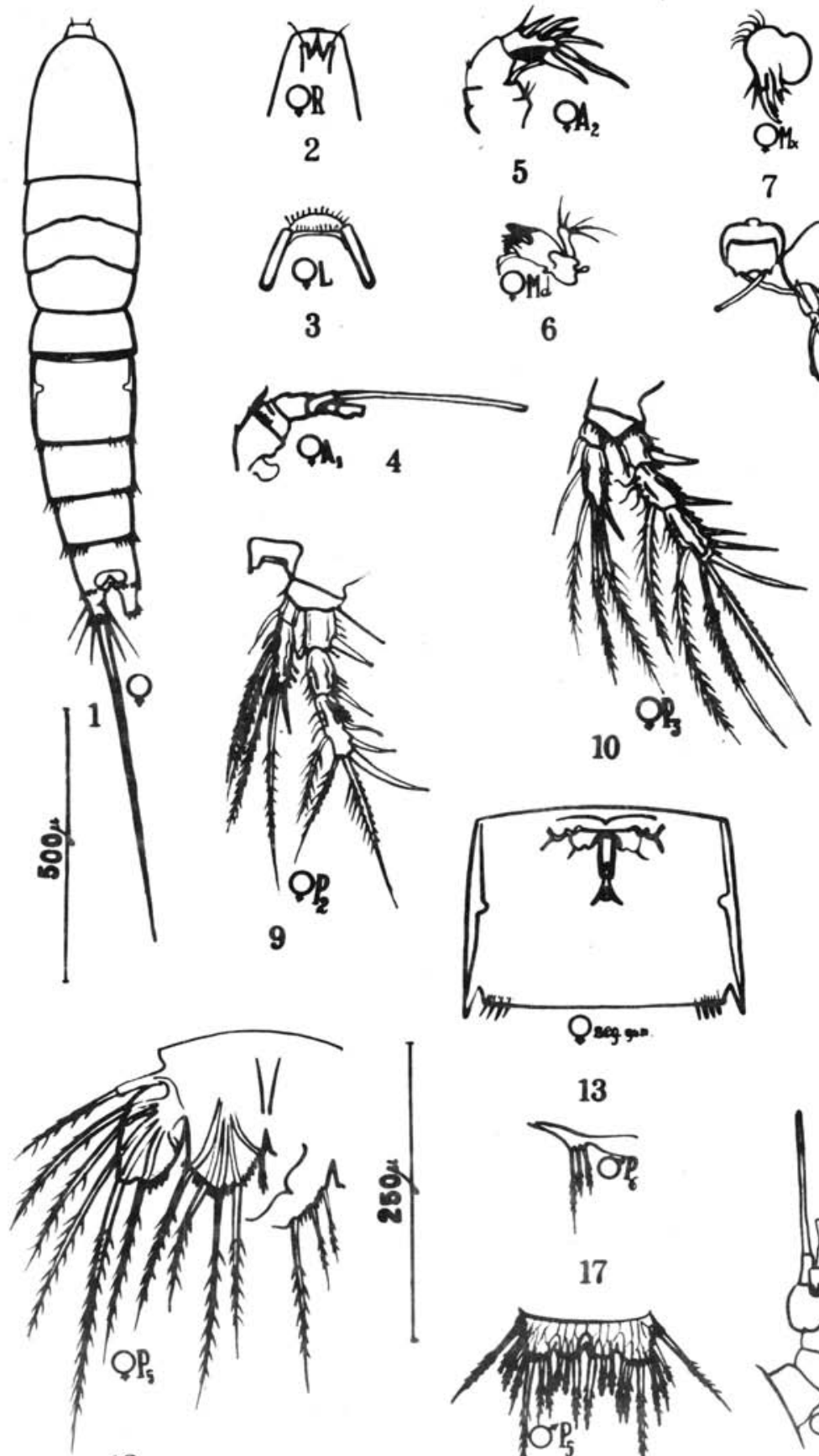

12

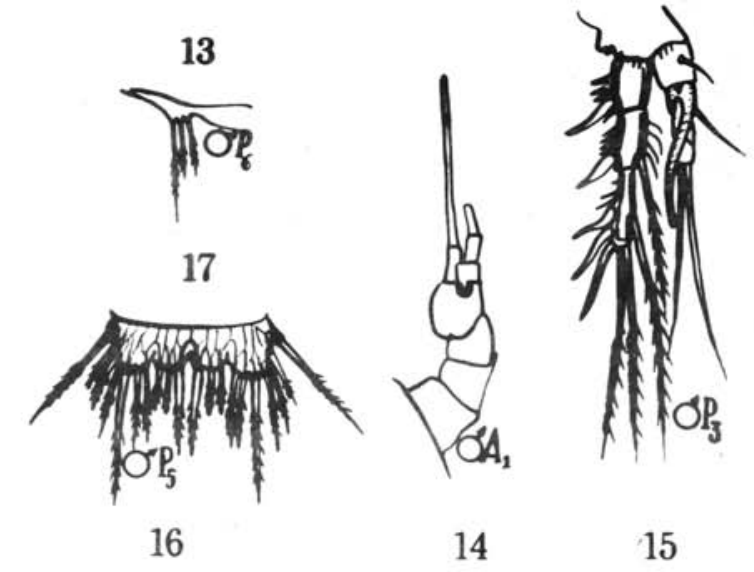




\section{ESTAMPA IV}

Katacletodes improportionatus n. Bp.

\begin{tabular}{|c|c|c|c|}
\hline Fig. & 1 & - & Viata dorsal \\
\hline$"$ & 2 & - & Antênula \\
\hline$\pi$ & 3 & - & Primeira perna \\
\hline ה & 4 & - & Segunda perna \\
\hline$"$ & 5 & - & Antena \\
\hline$n$ & 6 & - & Maxilipede \\
\hline ה & 7 & - & Terceira perna \\
\hline$\pi$ & 8 & - & Quarta perna \\
\hline$"$ & 9 & - & Mandíbula \\
\hline " & 10 & - & Segmento genital \\
\hline$"$ & 11 & - & Quinta perna \\
\hline
\end{tabular}


JakOH, H. - Esṕoles novas de Harpacticoida...

ESTAMPA IV
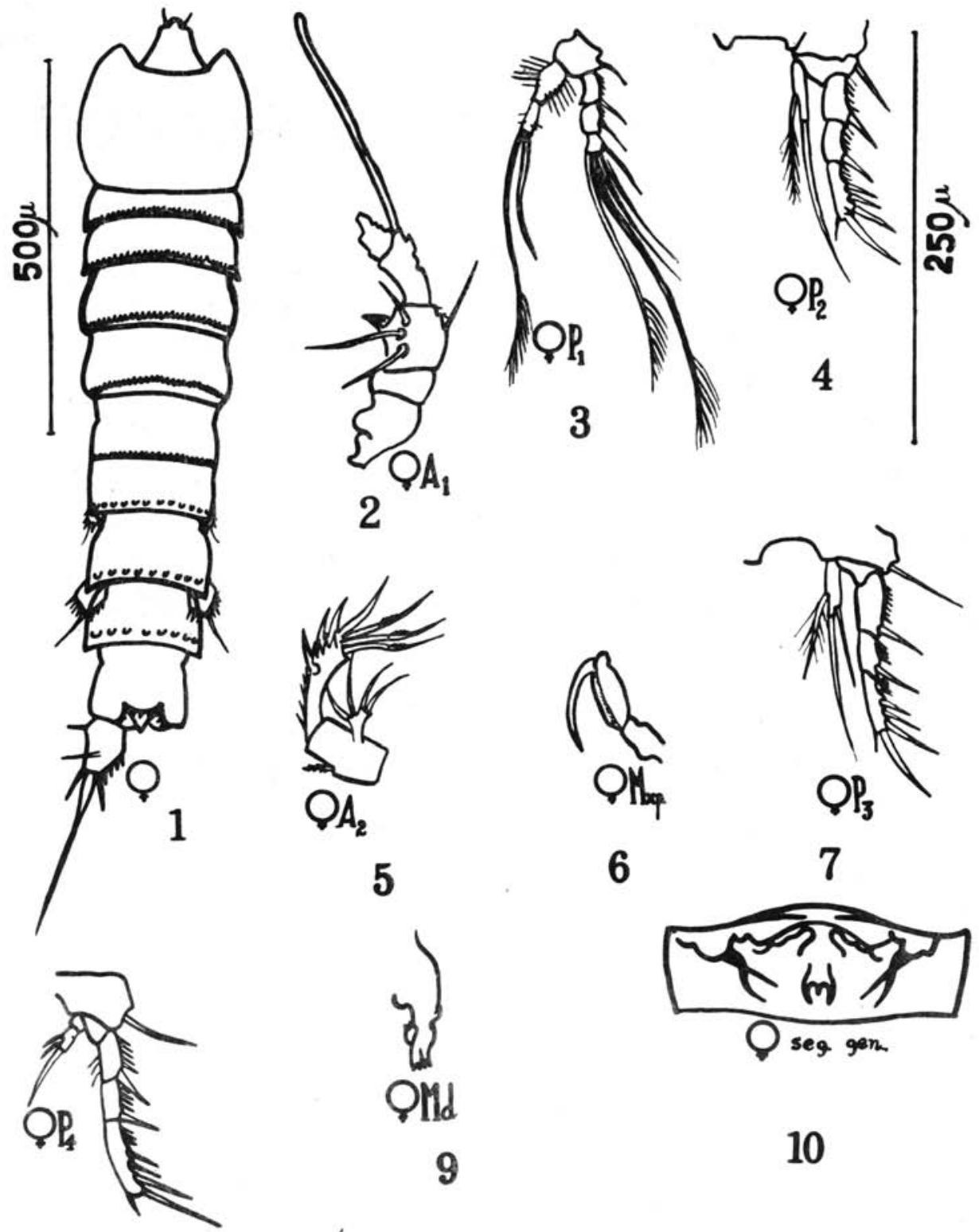

9
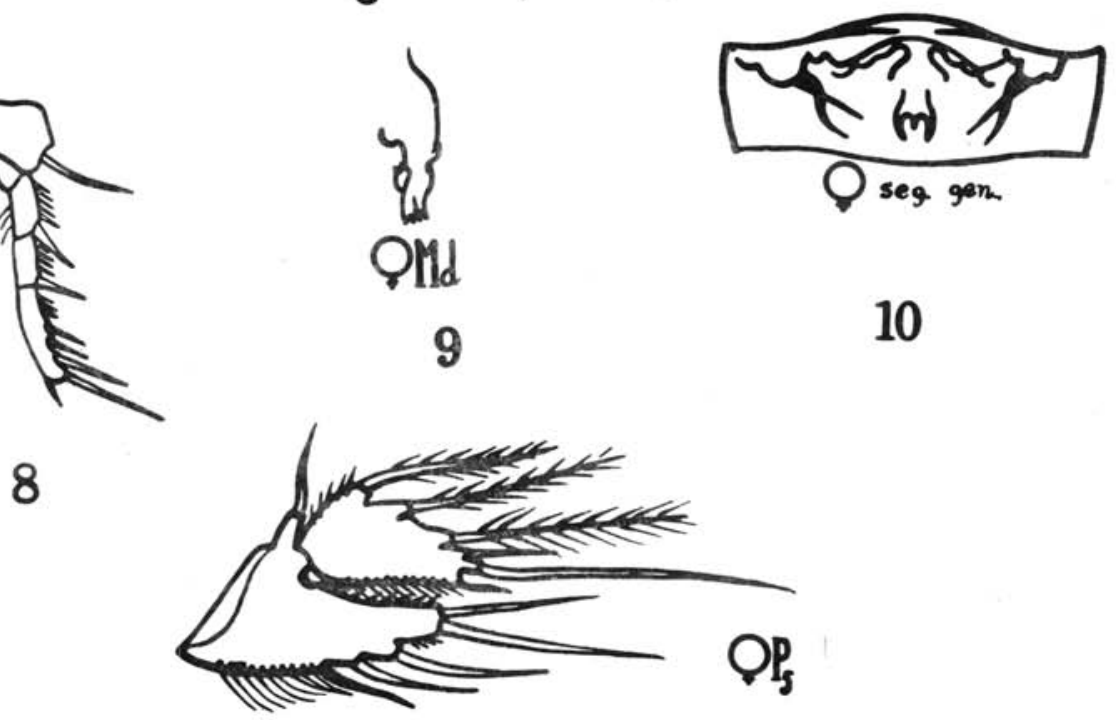


\section{ESTAMPA V}

Heterolaophonte manifera sulamericana nosubsp.

$\begin{array}{cll}\text { Fig. } & 1- & \text { Vista dorsal } \\ n & 2- & \text { Primeira perna } \\ n & 3- & \text { Segunda perna } \\ n & 4- & \text { Antena } \\ n & 5- & \text { Mandíbula } \\ n & 6- & \text { Maxilipede } \\ n & 7- & \text { Terceira perna } \\ n & 8- & \text { Segmento genital }\end{array}$

Heterolaophonte hamatus n.sp.

$\begin{array}{cl}\text { Fig. } 9- & \text { Primeira perna } \\ " 10- & \text { Quinta perna } \\ " 11- & \text { Antena } \\ \text { " } 12- & \text { Terceira perna }\end{array}$


JAKOBI, H. - Espété novas de Harpacticoida..

ESTAMPA V
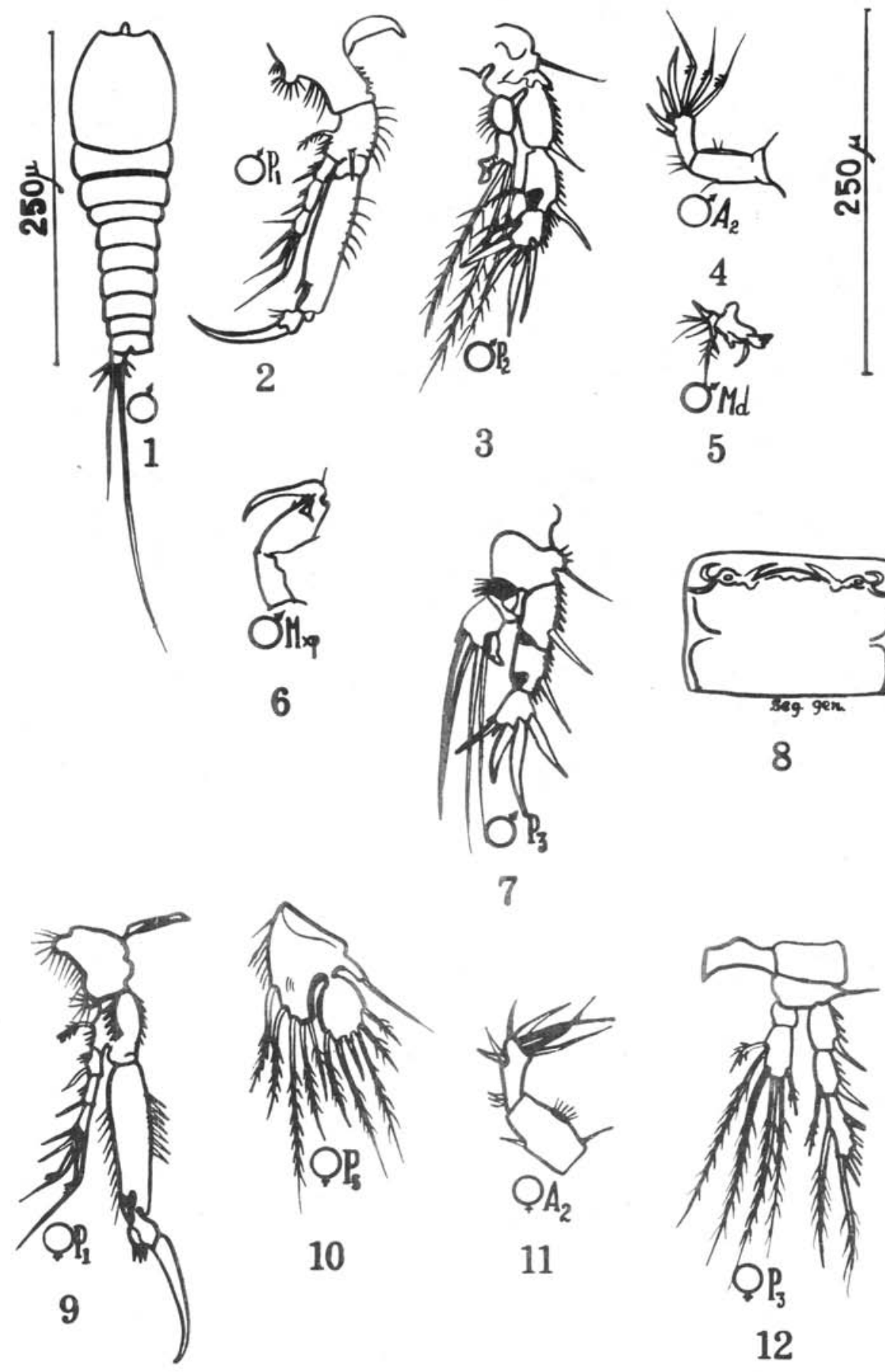

6
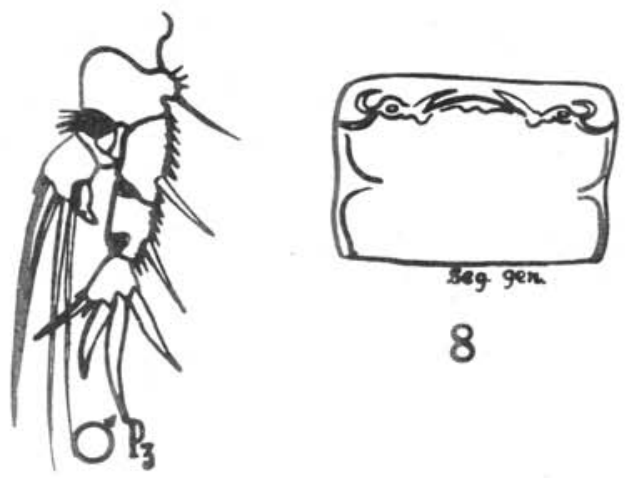

7
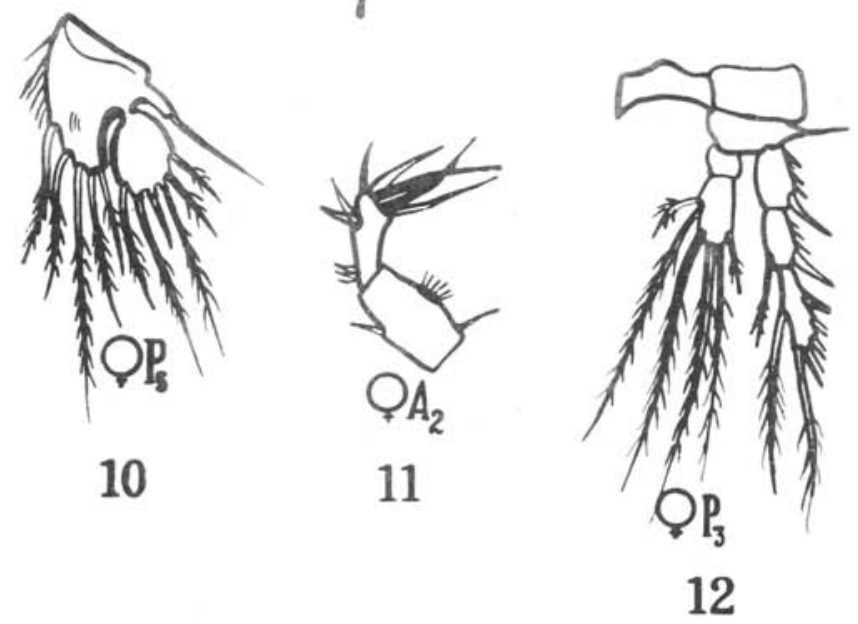
ESTAMPA VI

Onychocamptus besnardi n. sp.

\begin{tabular}{|c|c|c|c|}
\hline Fig. & 1 & - & Vista dorsal \\
\hline$n$ & 2 & - & Antênula e Rostrum \\
\hline$n$ & 3 & - & Primeira perna \\
\hline$n$ & 4 & - & Segunda perna \\
\hline$n$ & 5 & - & Quarta perna \\
\hline$n$ & 8 & - & Quinta perna \\
\hline " & 7 & - & Maxilípede \\
\hline " & 8 & - & Antena \\
\hline n & 9 & - & Antênula a \\
\hline " & 10 & - & Terceira perna \\
\hline$n$ & 11 & - & Quinta perna \\
\hline$n$ & 12 & - & Sexta perna \\
\hline$\pi$ & 13 & - & Jovens \\
\hline$\pi$ & 14 & & \\
\hline$\pi$ & 15 & - & Furca \\
\hline
\end{tabular}




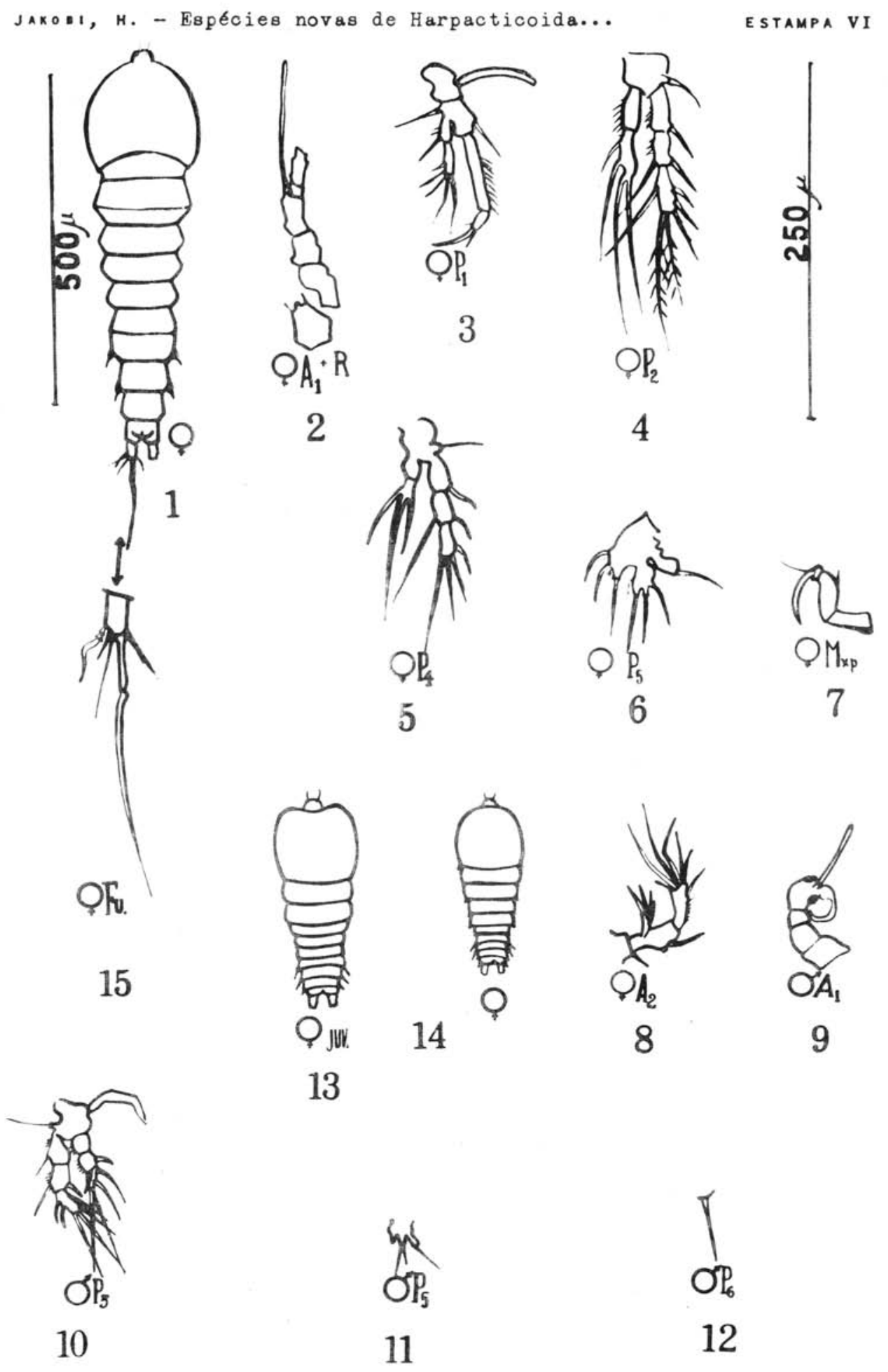

